\title{
Caracterização da fração volátil minoritária de bebida alcoólica fermentada de jabuticaba (Plinia jaboticaba) por CG/MS
}

\author{
Characterization of the minority volatile fraction of alcoholic fermented beverage from jabuticaba
}

(Plinia jaboticaba) by CG / MS

Caracterización de la fracción volátil minoritaria de bebida alcohólica fermentada de jabuticaba

(Plinia jaboticaba) por CG / MS

Recebido: 22/03/2021 | Revisado: 31/03/2021 | Aceito: 05/04/2021 | Publicado: 16/04/2021

\author{
Nathalia de Andrade Neves \\ ORCID: https://orcid.org/0000-0001-6936-2171 \\ Universidade Federal dos Vales do Jequitinhonha e Mucuri, Brasil \\ E-mail: nathalia.neves@ufvjm.edu.br \\ Paulo César Stringheta \\ ORCID: https://orcid.org/0000-0002-1229-7038 \\ Universidade Federal de Viçosa, Brasil \\ E-mail:p.stringheta@ufv.br
}

\begin{abstract}
Resumo
A composição volátil é de extrema importância na qualidade de bebidas alcoólicas, por influenciar diretamente no seu aroma. São escassos os estudos da composição volátil de bebidas pouco tradicionais, incluindo bebidas elaboradas a partir de jabuticaba. Este trabalho teve por objetivo a identificação dos compostos voláteis minoritários, por CG/MS, em fermentado alcoólico de jabuticaba. Foram identificados 78 compostos, dentre álcoois, ésteres, furanos, ácidos orgânicos, aldeídos e cetonas, acetatos, terpenos, compostos fenólicos voláteis e lactonas. A caracterização da fração volátil de fermentados alcoólicos de jabuticaba resultou em grande número de dados que, devido à sua extensão e ineditismo pode contribuir em trabalhos futuros que objetivem a melhoria da qualidade dessa bebida.
\end{abstract}

Palavras-chave: Myrciaria cauliflora; CG/MS; Sabará.

\begin{abstract}
The volatile composition is extremely important to the quality of alcoholic beverages, as it directly influences its aroma. There are few studies on the volatile composition of untraditional alcoholic beverages, including beverages made from jabuticaba. This work has the objective of identifying the minority volatiles compost, by CG / MS, in alcoholic fermented beverage from jabuticaba. Were identified 78 volatile compounds, among alcohols, esters, furans, organic acids, aldehydes and ketones, acetates, terpenes, volatile phenolic compounds and lactones. The characterization of the volatile fraction of alcoholic fermented beverage from jabuticaba resulted in a large number of data that, due to its extension and novelty, may contribute to future works that aim to improve the quality of this beverage.
\end{abstract}

Keywords: Myrciaria cauliflora; CG/MS; Sabará.

\section{Resumen}

La composición volátil es extremadamente importante en la calidad de las bebidas alcohólicas, ya que influye directamente en su aroma. Los estudios sobre la composición volátil de las bebidas alcohólicas no tradicionales son escasos, incluidas las bebidas elaboradas con jabuticaba. Este trabajo tuvo como objetivo identificar compuestos volátiles minoritarios, por CG/ MS, en fermentado alcohólico de jabuticaba. Se identificaron 78 compuestos, entre alcoholes, ésteres, furanos, ácidos orgánicos, aldehídos y cetonas, acetatos, terpenos, compuestos fenólicos volátiles y lactonas. La caracterización de la fracción volátil de lo fermentado alcohólico de jabuticaba resultó en una gran cantidad de datos que, por su extensión y novedad, pueden contribuir a futuros trabajos que tengan como objetivo mejorar la calidad de esta bebida.

Palabras clave: Myrciaria cauliflora; CG/MS; Sabará.

\section{Introdução}

Dentre as diversas variáveis que determinam a qualidade de uma bebida alcoólica, os aspectos sensoriais são os mais importantes do ponto de vista da aceitação do consumidor. Dentre elas, o aroma é o mais complexo e, consequentemente, o mais difícil de ser analisado. São mais de 800 compostos voláteis já descritos em diversos tipos de bebidas alcoólicas, entre 
álcoois, aldeídos, ésteres, cetonas, terpenos etc. Tais compostos possuem diferentes polaridades e volatilidades, além de estarem presentes em concentrações da ordem de ng a mg/L (Jiang \& Zhang, 2010; Pino \& Queris, 2011).

A importância no estudo dos aromas está, sobretudo, na diferenciação entre as bebidas, classificação da qualidade e detecção de pontos falhos do processo de produção, que incluem desde as etapas de fermentação até o armazenamento e que têm como consequência a formação de compostos indesejáveis às bebidas. Graças ao advento dos métodos cromatográficos, sobretudo os combinados com espectrometria de massa, a identificação e quantificação desses compostos tornou-se viável, mesmo para aqueles que estão contidos em pequenas frações (Nykänen, Suomalainen \& Suomalainen, 1983).

Em bebidas alcoólicas fermentadas, as leveduras são o principal agente de formação do aroma, que além do etanol e $\mathrm{CO}_{2}$, produzem inúmeras substâncias voláteis como co-produtos da fermentação, a partir de substratos como ácidos-graxos, compostos orgânicos nitrogenados, dentre outros (Tian et al., 2018). Dentre esses, os terpenos proporcionam aromas frutados e florais às bebidas. No entanto, os microrganismos também são responsáveis por diversos defeitos no aroma das bebidas, tais como acetatos, produzidos sobretudo por bactérias, metoxipirazinas, resultantes do metabolismo de aminoácidos, compostos sulfurados e elevadas concentrações de álcoois superiores (Carrau et al., 2008).

O fermentado alcoólico de jabuticaba é uma bebida tradicional no estado de Minas Gerais, onde é muito bem aceito (Neves, Fernandes, Valente, De Barros, \& Stringheta, 2020). No entanto são escassos os estudos sobre esse tipo de produto, não havendo trabalhos anteriores que explorem a composição de compostos voláteis minoritários em fermentados de jabuticaba (Neves et al., 2021). No intuito de contribuir para o conhecimento acerca desse produto, esse trabalho teve por objetivo a identificação de compostos voláteis minoritários em fermentados alcoólicos de jabuticaba produzidos artesanalmente.

\section{Metodologia}

\section{Elaboração dos fermentados alcoólicos}

A elaboração dos fermentados alcoólicos de jabuticaba ocorreu por meio do processo de fermentação em recipientes de vidro com capacidade nominal de $5 \mathrm{~L}$. Foram adicionados ao recipiente $1 \mathrm{~kg}$ de jabuticaba sabará (Plinia jaboticaba) esmagadas manualmente, 1,5 L de água mineral, 1,0 L de xarope contendo $700 \mathrm{~g}$ de sacarose e $0,5 \mathrm{~L}$ de uma suspensão contendo $10 \mathrm{~g}$ de leveduras liofilizadas (Saccharomyces cerevisae) da marca comercial Fleschmann. Após 72 horas do início do processo fermentativo, foi realizada a descuba, na qual as cascas e sementes de jabuticaba foram retirados do mosto de fermentação. Após o fim da fermentação, quando era observado o depósito de leveduras no fundo do recipiente, o fim da formação de bolhas de $\mathrm{CO} 2$ e a estabilização do teor de sólidos solúveis totais ( $\left.{ }^{\circ} \mathrm{Brix}\right)$, foi realizado o processo de trasfega, obtendo-se assim bebidas límpidas. $\mathrm{O}$ fermentado foi mantido sob refrigeração $\left(4^{\circ} \mathrm{C} \pm 1\right)$ por 7 dias, seguidos de uma segunda trasfega e envasamento em garrafas de vidro, de cor âmbar, com capacidade para 3,5 L. No momento do envase, foram adicionados $0,05 \mathrm{~g} / \mathrm{L}$ de metabissulfito de potássio com finalidade conservante. $\mathrm{O}$ fermentado foi elaborado em triplicata.

A análise da composição volátil ocorreu após seis meses de envelhecimento do fermentado.

\section{Preparo das amostras - extração em fase sólida}

Para a análise cromatográfica, foi necessária a etapa de extração das amostras, realizada tomando-se $25 \mathrm{~mL}$ de fermentado e adicionando $25 \mathrm{~mL}$ de água e $500 \mu \mathrm{L}$ da solução de 4 -nonanol $(0,1 \mathrm{~g} / \mathrm{L})$ utilizada como padrão interno à amostra. Foram utilizados cartuchos para extração em fase sólida LiChrolut EN $(40-120 \mu \mathrm{m})$ de $5 \mathrm{~mL}$ com 0,2 g de adsorvente. Primeiramente, os cartuchos foram acondicionados com $2 \mathrm{~mL}$ de pentano:diclorometano 2:1, seguidos de $2 \mathrm{~mL}$ de metanol P.A. e $5 \mathrm{~mL}$ de etanol $6 \%(\mathrm{v} / \mathrm{v})$. Após o acondicionamento foi introduzida a amostra, gota a gota pelo cartucho. Os compostos polares foram extraídos com $25 \mathrm{~mL}$ de água e em seguida foi realizada a extração dos compostos voláteis minoritários por 
meio da passagem pelo cartucho de $15 \mathrm{~mL}$ de pentano:diclorometano. A porção recuperada foi mantida em congelador por 24 h, após as quais foi descartada a porção que se manteve congelada e se recuperou a porção líquida. O material recuperado foi ainda adicionado de $500 \mu \mathrm{L}$ de etanol absoluto e em seguida foi submetido à concentração por jato de nitrogênio. As amostras obtidas ao final do processo foram levadas à análise cromatográfica.

\section{Análise dos compostos voláteis por CG/MS}

Os compostos voláteis foram analisados por cromatografia gasosa acoplada a espectroscopia de massa (GC/MS) ThermoQuest GC2000 e detector de massa DSQII quadruplo. A metodologia descrita por Cañas, Pérez-Martín, Romero, Prieto, e Herreros, (2012) foi utilizada como referência com algumas modificações. Foi utilizada coluna BP21 de fase estacionaria tipo FFAP com $60 \mathrm{~m}$ de longitude e $0,32 \mathrm{~mm}$ de diâmetro. O gás utilizado com fase móvel foi o Hélio $(1,4$ $\mathrm{mL} / \mathrm{min}$ Split 1/15) à temperatura de injeção de $210{ }^{\circ} \mathrm{C}$ e condições de temperatura do forno: $45{ }^{\circ} \mathrm{C}(15 \mathrm{~min})-2{ }^{\circ} \mathrm{C} / \mathrm{mim}, 100$ ${ }^{\circ} \mathrm{C}-1{ }^{\circ} \mathrm{C} / \mathrm{min}, 150{ }^{\circ} \mathrm{C}-4{ }^{\circ} \mathrm{C} / \mathrm{min}, 210{ }^{\circ} \mathrm{C}(45 \mathrm{~min})$. O volume de injeção foi de $2 \mu \mathrm{L}$ das amostras previamente extraídas em fase sólida.

$\mathrm{O}$ detector foi operado a uma voltagem $1250 \mathrm{~V}$ com temperatura da fonte de $250{ }^{\circ} \mathrm{C}$, temperatura da interface $\mathrm{GC}$ de $250^{\circ} \mathrm{C}$, corrente de emissão de $150 \mu \mathrm{V}$ e energia de impacto de $70 \mathrm{eV}$. Os compostos separados foram identificados com base na espectroscopia de massa e tempo de retenção, utilizando-se padrões comerciais como referência. A quantificação de alguns compostos foi realizada por meio da análise das características de fragmentação de cada composto tendo como referência o padrão interno, sendo que cada composto teve a sua linearidade verificada conforme referência (Izquierdo- Cañas, GarcíaRomero, Gómez-Alonso, \& Palop Herreros, 2008).

\section{Resultados e Discussão}

Foi identificado um total de 78 compostos voláteis minoritários na bebida alcoólica fermentada de jabuticaba, pertencentes às classes de álcoois, ésteres, furanos, ácidos orgânicos, aldeídos e cetonas, acetatos, terpenos, compostos fenólicos voláteis e lactonas (Tabela 1).

Tabela 1. Compostos voláteis minoritários identificados em bebida alcoólica fermentada de jabuticaba produzido artesanalmente. Concentração em $\mu \mathrm{g} / \mathrm{L}$.

\begin{tabular}{cc}
\hline Composto & Concentração Encontrada \\
\hline 2-pentanol & Álcool \\
4-metil-1-pentanol & $1459,376 \pm 396,751$ \\
3-metil-1-pentanol & $14,483 \pm 6,063$ \\
3-metil-3-buten-1-ol & $66,083 \pm 26,487$ \\
3-etoxi-1-propanol & $14,789 \pm 8,090$ \\
3-octanol & $17,923 \pm 4,581$ \\
1-heptanol & $0,610 \pm 0,067$ \\
2-etil-1-hexanol & $7,758 \pm 0,670$ \\
1-octanol & $34,819 \pm 5,813$ \\
1-hexanol & $3,651 \pm 1,549$ \\
c-3-hexen-1-ol & $7,011 \pm 2,146$ \\
t-2-hexenol & $23,266 \pm 2,104$ \\
Álcool benzílico & $126,385 \pm 4,973$ \\
2- feniletanol & $8,220 \pm 1,755$ \\
3-metil-tio-propanol & $96201,803 \pm 8729,340$ \\
3,3-dietoxi-1-propanol & $510,782 \pm 15,328$ \\
1-butanol & n.q. \\
4-nonanol & n.q. \\
t-nerolidol & n.q \\
t-cadinol & n.q \\
& n.d \\
\hline
\end{tabular}




\begin{tabular}{|c|c|}
\hline \multicolumn{2}{|c|}{ Ésteres } \\
\hline Hexanoato de etila & $148,148 \pm 26,294$ \\
\hline Etil-2-butenoato & $45,071 \pm 5,679$ \\
\hline Etil-2-OH-3-metil-butirato & $49,875 \pm 16,322$ \\
\hline Octanoato de etila & $20,516 \pm 6,027$ \\
\hline 3-OH-butirato de etila & $167,986 \pm 74,912$ \\
\hline Etil-2-OH-hexanoato & $273,352 \pm 52,433$ \\
\hline 4-oxo-butanoato de etila & $13,974 \pm 1,130$ \\
\hline 2-furoato de etila & $8,871 \pm 2,164$ \\
\hline Decanato de etila & $2,523 \pm 0,472$ \\
\hline Succinato de dietila & $377,195 \pm 32,844$ \\
\hline 2,7,dimetil-4,5-octanodiol & $129,807 \pm 57,206$ \\
\hline Benzoacetato de etila & $4,458 \pm 0,582$ \\
\hline 4-OH-butanoato-de-etila & $5512,058 \pm 3044,538$ \\
\hline Dodecanoato de etila & $6,550 \pm 1,866$ \\
\hline Malonato de dietila & $158,464 \pm 63,471$ \\
\hline Hexadecanoato de etila & $37,236 \pm 6,796$ \\
\hline Monosuccinato de etila & $43,947 \pm 13,748$ \\
\hline Fenetil-lactato & $375,427 \pm 48,281$ \\
\hline Malonato de dimetila & $8,678 \pm 2,753$ \\
\hline 4-metil-2-penteno & $74,511 \pm 4,441$ \\
\hline 4-OH-etil-ácido-benzenoacético & $147,620 \pm 16,407$ \\
\hline Diethyl-2-metilenesuccinato & n.q. \\
\hline 3-metil-butirato de etila & n.q. \\
\hline butanoato de isoamilo & n.q. \\
\hline lactato de isoamilo & n.q. \\
\hline \multicolumn{2}{|c|}{ Furanos } \\
\hline Furfural & $3,195 \pm 1,141$ \\
\hline 5-hidroximetil-furfural (HMF) & $4,507 \pm 1,885$ \\
\hline \multicolumn{2}{|l|}{ - } \\
\hline Ácido isobutírico & $865,678 \pm 44,976$ \\
\hline ácido butírico & $116,803 \pm 56,846$ \\
\hline Ácido isovalérico & $4048,791 \pm 350,501$ \\
\hline Ácido octanoico & $3307,597 \pm 188,024$ \\
\hline Ácido decanóico & $210,763 \pm 73,425$ \\
\hline Ácido fenilacético & $10,000 \pm 3,108$ \\
\hline Ácido hexanóico & $1959,275 \pm 809,322$ \\
\hline \multicolumn{2}{|c|}{ Aldeídos e cetonas } \\
\hline Benzaldeído & $6,373 \pm 0,488$ \\
\hline Acetofenona & $24,101 \pm 0,307$ \\
\hline Siringaldeído & $4,384 \pm 0,714$ \\
\hline Vanilina & $1,488 \pm 0,891$ \\
\hline Acetovanilona & $1,314 \pm 0,552$ \\
\hline \multicolumn{2}{|c|}{ Acetatos } \\
\hline Acetato de isobutilo & $1,190 \pm 0,783$ \\
\hline Acetato isoamílico & $442,464 \pm 67,701$ \\
\hline Acetato de 1,3,-propanodiol & $18,316 \pm 3,513$ \\
\hline 2-feniletil-acetato & $304,755 \pm 49,631$ \\
\hline \multicolumn{2}{|c|}{ Terpenos } \\
\hline Linalol & $21,619 \pm 1,711$ \\
\hline$\alpha$-terpineol & $19,409 \pm 1,092$ \\
\hline Eucaliptol & n.q \\
\hline \multicolumn{2}{|c|}{ Fenólicos voláteis } \\
\hline Fenol & $5,147 \pm 1,958$ \\
\hline 4-etil-guaiacol & $0,194 \pm 0,113$ \\
\hline 4-vinil-guaiacol & $9,468 \pm 0,909$ \\
\hline Guaiacol & $7,259 \pm 0,456$ \\
\hline Siringol & $8,461 \pm 1,098$ \\
\hline Tirosol & $3360,722 \pm 385,790$ \\
\hline 3,4-dimetoxi-fenol & n.q \\
\hline
\end{tabular}




\begin{tabular}{cc}
\hline & Lactonas \\
$\gamma$-butirolactona & $72,704 \pm 21,547$ \\
$\gamma$-nanolactona & $1,577 \pm 0,571$ \\
Pantolactona & $4,660 \pm 1,437$ \\
$\gamma$-etoxicarbonil- $\gamma$-butirolactona & $538,046 \pm 45,430$ \\
4-etoxi-butirolactona & n.q \\
\hline
\end{tabular}

N.q.= valores não quantificados. Fonte: Autores.

Os ésteres e álcoois foram as classes de compostos mais abundantes nos fermentados alcoólicos de jabuticaba, ambos resultantes do processo fermentativo. Os ésteres são importantes componentes do aroma, responsáveis pelas notas de frescor e frutadas em vinhos. Os álcoois superiores, por sua vez, são essencialmente formados pelo catabolismo do açúcar ou pela descarboxilação e desaminação de aminoácidos e têm forte influência no sabor das bebidas (Li et al., 2017).

Os álcoois estão presentes nas bebidas alcoólicas como resultado do metabolismo de leveduras a partir da degradação de carboidratos, aminoácidos e lipídeos. Os álcoois superiores, provenientes principalmente da transformação de aminoácidos, são formados nos primeiros dois estágios da fermentação alcoólica. Em pequenas concentrações, contribuem para o aroma frutado das bebidas. Concentrações elevadas (mais de $100 \mathrm{mg} / 100 \mathrm{~mL}$ ) exercem efeitos contrários, além de causar toxidez com sintomas como náuseas e dores de cabeça (Zhong et al., 2020). No fermentado alcoólico de jabuticaba, os álcoois presentes em concentrações mais elevadas foram o 2-pentanol e o 2-feniletanol, além de t-2-hexenol e 3-metil-tio-propranol. O 2-feniletanol é um álcool superior de ampla ocorrência na natureza, sendo associado ao aroma de rosas e muito comum em vinhos de uvas, estando presentes nessas bebidas em concentrações relativamente altas. (Cardeal, Souza, Silva \& Marriott, 2008).

O 2-pentanol, ou álcool sec-amílico, embora tenha sido encontrado em concentrações elevadas no fermentado, não é muito comum em vinhos de uva, estando presentes apenas em traços nessas bebidas. Em trabalho sobre a elaboração de bebidas destiladas a partir da jabuticaba (Asquieri, Silva \& Cândido, 2009) foi detectada a presença de álcool amílico em concentrações na ordem $156 \mathrm{mg} / 100 \mathrm{~mL}$. Ainda segundo os mesmos autores, o álcool amílico tem ação solvente sobre outras substâncias aromáticas interferindo nos graus de volatilidade destas.

Em trabalho anterior com fermentado alcoólico de jabuticaba, foi reportada a presença dos álcoois 2-pentanol $(3,1 \pm$ 0,4 $\mu \mathrm{g} / \mathrm{L}), 1$-butanol (15,6 \pm 1,5 $\mu \mathrm{g} / \mathrm{L}), 4$-metil-1-pentanol (6,3 \pm 0,5 $\mu \mathrm{g} / \mathrm{L}), 3$-metil-1-pentanol (13,7 \pm 0,4 $\mu \mathrm{g} / \mathrm{L}), 3$-etoxi-1propanol $(30,6 \pm 0,0 \mu \mathrm{g} / \mathrm{L})$, 1-heptanol $(2,3 \pm 0,7 \mu \mathrm{g} / \mathrm{L}), 2$-etil-1-hexanol $(12,6 \pm 0,8 \mu \mathrm{g} / \mathrm{L})$, e álcool benzílico $(17,2 \pm 0,7$ $\mu \mathrm{g} / \mathrm{L}$ ) em comum com esse trabalho (Duarte et al., 2010). De todos os álcoois encontrados, somente o álcool benzílico estava presente em maiores concentrações no trabalho desses autores. O álcool 1-butanol não foi quantificado nesse trabalho, não havendo meios para a comparação. Esta diferença encontrada entre os dois trabalhos é esperada, uma vez que os demais álcoois produzidos pelas leveduras, em especial os álcoois superiores, são formados a partir de mecanismos complexos, que envolvem vias catabólicas e anabólicas, sendo resultantes do processo metabólico de leveduras a partir de aminoácidos. A quantidade de álcoois superiores produzidos depende da cepa e espécie da levedura, quantidade de inóculo utilizado, temperatura na qual ocorreu a fermentação e composição do meio (Pietruszka, Pielech-Przybylska, \& Szopa, 2010).

Os ésteres em geral são desejáveis às bebidas alcoólicas por contribuírem com o buquê das bebidas, proporcionando notas para o aroma frutado (Izquierdo-Cañas et al., 2012). Eles ocorrem naturalmente nas frutas, além de serem formados pelas leveduras durante a fermentação alcoólica. Em especial, os etil-ésteres, são formados pela quebra do etanol durante a síntese ou degradação de lipídeos (Juan, Jianquan, Junni, Zijian, \& Ji, 2012).

O principal éster identificado no fermentado foi o 4-OH-butanoato de etila, característico do aroma de abacaxi e que foi detectado em concentrações de 14,6 a 2780 vezes superior aos demais ésteres. Presente em diversas bebidas alcoólicas fermentadas, esse também foi o principal éster encontrado em suco e vinhos elaborados a partir da variedade de uva Madras (Kamaleddin et al., 2020). 
Os compostos pertencentes à classe dos furanos são formados a partir da termodegradação de carboidratos por caramelização e decomposição de polissacarídeos (Pino \& Queris, 2011). Dentre os furanos, foram encontrados no fermentado alcoólico de jabuticaba somente furfural e 5-hidroximetil-furfural (HMF). O HMF é um aldeído heterocíclico solúvel em água que está comumente presente em nível baixo em alimentos frescos contendo açúcares (Ortu \& Caboni, 2017). Apesar de presentes, esses compostos exercem pouco efeito sob o aroma final do fermentado, devido à baixa concentração.

Foram encontrados sete diferentes ácidos orgânicos voláteis, sendo que os ácidos isovalérico, octanoico e hexanóico estavam presentes em concentrações superiores a $1000 \mu \mathrm{g} / \mathrm{L}$. Esses ácidos são comumente encontrados em vinhos, produzidos pelas leveduras durante a fermentação alcoólica (Robles, Fabjanowicz, Chmiel, \& Płotka-Wasylka, 2019).

Em baixas concentrações, os aldeídos e cetonas podem ocasionar em aromas frutais ou florais em vinhos. Porém altas concentrações podem ser indicadores de deterioração causada por aquecimento ou contaminação e podem levar a sabores amargos ou rançosos. Em concentrações ainda mais elevadas, podem causar toxidez e irritações (Stockton et al., 2010).

$\mathrm{O}$ acetaldeído, muito comum em bebidas alcoólicas, podendo ser encontrado em concentrações ente 400 e $600 \mu \mathrm{M}$ em vinhos, não foi detectado no fermentado alcoólico de jabuticaba. Este composto está associado à oxidação dos vinhos além de promover a rápida polimerização das antocianinas, catequinas e taninos (Osborne, Orduña, Polone \& Liu, 2000).

A acetofenona foi a cetona mais abundante no fermentado. Típico de vinhos envelhecidos, esse composto está associado a aromas frutados (Wang, Chen, \& Zhou, 2020).

Durante a fermentação alcoólica, muitos ésteres são formados em decorrência do metabolismo microbiano. Dentre eles, os acetatos estão presentes em maiores concentrações e contribuem de maneira importante para o aroma de vinhos tradicionais (Plata, Millan, Mauricio \& Ortega, 2003). A produção de ésteres de acetato depende do balanço entre a síntese de ésteres por álcool acetiltransferase e a hidrólise por ester-hidrolases. Está diretamente relacionado às cepas das leveduras (Saccharomyces cereviseae) utilizadas, sendo que alguns autores sugerem que a utilização de outras espécies de leveduras pode incrementar o aroma de vinhos (Rojas, Gil, Piñaga \& Manzanares, 2003).

O fermentado alcoólico de jabuticaba apresentou como acetatos majoritários o acetato isoamílico e o 2-fenoletil. O acetato isoamílico é um dos ésteres que mais contribuem para o perfil do aroma de vinhos brancos (Plata et al., 2003), proporcionando um aroma característico de banana. Por sua vez, o acetato 2-feniletil proporciona aromas frutados e florais, com notas de mel (Rojas et al., 2003).

Os terpenos são uma extensa classe de compostos de baixo peso molecular e de larga distribuição na natureza. Em bebidas alcoólicas, contribuem positivamente para o aroma. Estão presentes nos vinhos, sendo que podem ser originários da própria uva ou produzidos por enzimas durante o processo de fermentação ou de maturação das bebidas. Já foram identificados também em cachaças e outras bebidas destiladas (Cardeal \& Marriott, 2010). Podem ser encontrados nas formas livre ou combinada com glicosídeos, sendo que a forma combinada não apresenta o aroma desejado. A concentração na forma livre é resultante da atividade da enzima $\beta$-glicosidase das leveduras, que hidrolisa aqueles presentes na forma glicosilada e aumenta o teor de terpenos aromáticos nas bebidas (Izquierdo-Cañas et al., 2012). As leveduras S. cerevisiae, segundo alguns autores, não apresentam atividade de $\beta$-glicosidase, justificando a baixa concentração de terpenos livres em vinhos (Maicas \& Mateo, 2005).

Foram encontrados três tipos de terpenos no fermentado alcoólico de jabuticaba, sendo eles, linalol, $\alpha$-terpineol e eucaliptol. O linalol possui perfume floral que remete a condimentos e limão e é encontrado em uvas e vinhos. Possui limite de detecção olfativa baixo $(6 \mu \mathrm{g} / \mathrm{L})$, sendo facilmente detectado (Teixeira, Marques, Rocha, \& Moreira, 2020). O $\alpha$-terpineol também é encontrado em uvas e possui aroma que remete ao melão. No entanto, seu limite de detecção olfativa é cerca de 4 a 10 vezes menor do que o linalol (330-353 $\mu \mathrm{g} / \mathrm{L}$ ), havendo maiores dificuldades de detecção pelo olfato humano (Lengyel, 2012). 
Os fenólicos voláteis presentes em bebidas alcoólicas são normalmente produzidos a partir do metabolismo das leveduras e podem, muitas vezes, transmitir odores e sabores desagradáveis (Cabrita, Palma, Patão \& Freitas, 2012). Em vinhos, esta classe de compostos causa sensações de amargor e adstringência além de influenciar na percepção de doçura e acidez e exercer impacto na percepção do corpo (Jackson, 2009). No entanto, alguns desses fenólicos possuem atividades antioxidante e antiflamatória. Dentre os fenólicos encontrados no fermentado alcoólico de jabuticaba, o tirosol apresentou concentração maior do que dos demais. Esse é um fenólico abundante em vinhos, cervejas e em azeite de oliva, com reconhecida atividade antioxidante (Souza, Vásquez, Mastro, Acree \& Lavin, 2006). Em vinhos, concentrações acima de 25 $\mathrm{mg} / \mathrm{L}$ causam amargor nas bebidas. A concentração encontrada nesse trabalho não é suficiente para causar esse efeito.

As lactonas são amplamente distribuídas na natureza, sobretudo em frutos como damasco, pêssego e morango. Em vinhos, são produzidas a partir da fermentação, esterificação intra-molecular dos ácidos hidroxicinamicos correspondentes ou são liberados pela madeira dos barris que armazenam a bebida (Liberatore, Pati, Nobile, \& Notte, 2010), contribuindo para o aroma frutado (Pino \& Queris, 2011). Os fermentados de jabuticaba apresentaram algumas lactonas que, como essa bebida não foi envelhecida em barris, são provavelmente resultantes do processo fermentativo.

\section{Conclusão}

Foram identificados voláteis originários da transformação de compostos naturalmente presentes na jabuticaba, além de compostos originados em decorrência do processo de fermentação alcoólica.

Os compostos encontrados são caraterísticos de bebidas alcoólicas, sobretudo vinhos, devido à semelhança entre dos processos de obtenção dos vinhos e fermentados alcoólicos de frutas. Não foram encontrados compostos reconhecidos por causar efeitos indesejáveis ao sabor e aroma das bebidas, em concentrações suficientes para tal.

A caracterização da fração volátil de fermentados alcoólicos de jabuticaba resultou em grande número de dados que, devido à sua extensão e ineditismo pode contribuir em trabalhos futuros que objetivem a melhoria da qualidade dessas bebidas.

\section{Referências}

Asquieri, E. R., Silva, A. G. de M., \& Cândido, M. A. (2009). Aguardente de jabuticaba obtida da casca e borra da fabricação de fermentado de jabuticaba. Ciência e Tecnologia de Alimentos, 29(4), 896-904.

Cabrita, M. J., Palma, V., Patão, R., \& Freitas, A. M. C. (2012). Conversion of hydroxycinnamic acids into volatile phenols in a synthetic medium and in red wine by Dekkera bruxellensis. Ciência e Tecnologia de Alimentos, 32(1), 106-112. https://doi.org/10.1590/S0101-20612012005000024

Cañas, P. M. I., Pérez-Martín, F., Romero, E. G., Prieto, S. S., \& Herreros, M. de los L. P. (2012). Influence of inoculation time of an autochthonous selected malolactic bacterium on volatile and sensory profile of Tempranillo and Merlot wines. International Journal of Food Microbiology, 156(3), 245-254. https://doi.org/10.1016/j.ijfoodmicro.2012.03.033

Cardeal, Z. de L., \& Marriott, P. J. (2010). Caracterização das Etapas de Produção de Cachaça por meio de Perfis Cromatográficos Obtidos por Cromatografia Gasosa Bidimensional Abrangente. Scientia Chromatographica, 2(3), 69-80.

Cardeal, Z. L., de Souza, P. P., Silva, M. D. R. G. da., \& Marriott, P. J. (2008). Comprehensive two-dimensional gas chromatography for fingerprint pattern recognition in cacha??a production. Talanta, 74(4), 793-799. https://doi.org/10.1016/j.talanta.2007.07.021

Duarte, W. F., Dias, D. R., Oliveira, J. M., Teixeira, J. A., de Almeida e Silva, J. B., \& Schwan, R. F. (2010). Characterization of different fruit wines made from cacao, cupuassu, gabiroba, jaboticaba and umbu. LWT - Food Science and Technology, 43(10), 1564-1572. https://doi.org/10.1016/j.1wt.2010.03.010

Jackson, R. S. (2009). Wine Tasting: A Professional Book.

Juan, C., Jianquan, K., Junni, T., Zijian, C., \& Ji, L. (2012). The Profile in Polyphenols and Volatile Compounds in Alcoholic Beverages from Different Cultivars of Mulberry. Journal of Food Science, 77(4), 430-436. https://doi.org/10.1111/j.1750-3841.2011.02593.x

Kamaleddin, F. H., Fikrat, I. K., Elman, H. E., Telman, I. M., Musa, M. T., Ramiz, A. G., \& Hafiz, H. S. (2020). A Study of the fragrances in the pink juice and wines from grapes madras. Sylwan, 164(7), 62-68.

Lengyel, E. (2012). Primary aromatic character of wines. Acta Universitatis Cibiniensis, Series E: Food Technology, XVI(1), 3-18.

Li, S., An, Y., Fu, W., Sun, X., Li, W., \& Li, T. (2017). Changes in anthocyanins and volatile components of purple sweet potato fermented alcoholic beverage during aging. Food Research International, 100, 235-240. https://doi.org/10.1016/j.foodres.2017.08.041 
Liberatore, M. T., Pati, S., Nobile, M. A. Del., \& Notte, E. La. (2010). Aroma quality improvement of Chardonnay white wine by fermentation and ageing in barrique on lees. Food Research International, 43(4), 996-1002. https://doi.org/10.1016/j.foodres.2010.01.007

Maicas, S., \& Mateo, J. J. (2005). Hydrolysis of terpenyl glycosides in grape juice and other fruit juices: a review. Applied microbiology and biotechnology, 67, 322-335.

Neves, N. de A., Stringueta, P. C., García-Romero, E., \& Gómez-Alonso, S. (2021). Elaboração e caracterização de fermentado alcoólico de jabuticaba (Plinia jaboticaba) produzido artesanalmente. Research, Society and Development, 10(4), 1-12. https://doi.org/10.33448/rsd-v10i4.13799

Ortu, E., \& Caboni, P. (2017). Levels of 5-hydroxymethylfurfural, furfural, 2-furoic acid in sapa syrup, Marsala wine and bakery products. International Journal of Food Properties, 20(sup3), S2543-S2551. https://doi.org/10.1080/10942912.2017.1373668

Pietruszka, M., Pielech-Przybylska, K., \& Szopa, J. S. (2010). Synthesis of higher alcohols during alcoholic fermentation of rye mashes. Scientific Bulletin of the Technical University of Lodz, 74(1081), 51-64.

Pino, J. A., \& Queris, O. (2011). Analysis of volatile compounds of mango wine. Food Chemistry, 125, 1141-1146.

Plata, C., Millan, C., Mauricio, J. C., \& Ortega, J. M. (2003). Formation of ethyl acetate and isoamyl acetate by various species of wine yeasts. Food Microbiology, 20, 217-224.

Robles, A., Fabjanowicz, M., Chmiel, T., \& Płotka-Wasylka, J. (2019). Determination and identification of organic acids in wine samples. Problems and challenges. TrAC Trends in Analytical Chemistry, 120, 115630. https://doi.org/10.1016/j.trac.2019.115630

Rojas, V., Gil, J. V., Piñaga, F., \& Manzanares, P. (2003). Acetate ester formation in wine by mixed cultures in laboratory fermentations. International Journal of Food Microbiology, 86, 181-188.

Souza, M. D. C. A. de., Vásquez, P., Mastro, N. L. del, Acree, T. E., \& Lavin, E. H. (2006). Characterization of Cachaça and Rum Aroma. Journal of Agricultural and Food Chemistry, 54(2), 485-488. https://doi.org/10.1021/jf0511190

Stockton, A. M., Tjin, C. C., Huang, G. L., Benhabib, M., Chiesl, T. N., \& Mathies, R. A. (2010). Analysis of carbonaceous biomarkers with the Mars Organic Analyzer microchip capillary electrophoresis system: Aldehydes and ketones. Electrophoresis, 31(22), 3642-3649. https://doi.org/10.1002/elps.201000424

Teixeira, I. E. A. Z., Marques, T. S., Rocha, C. B., \& Moreira, R. F. A. (2020). A fração volátil da camomila alemã (Matricaria recutita L.). Research, Society and Development, 9(7), e18973510. https://doi.org/10.33448/rsd-v9i7.3510

Tian, T., Yang, H., Yang, F., Li, B., Sun, J., Wu, D., \& Lu, J. (2018). Optimization of fermentation conditions and comparison of flavor compounds for three fermented greengage wines. $L W T, 89,542-550$.

Wang, N., Chen, S., \& Zhou, Z. (2020). Age-dependent characterization of volatile organic compounds and age discrimination in Chinese rice wine using an untargeted GC/MS-based metabolomic approach. Food Chemistry, 325, 126900.

Zhong, X., Wang, A., Zhang, Y., Wu, Z., Li, B., Lou, H., \& Wen, H. (2020). Reducing higher alcohols by nitrogen compensation during fermentation of Chinese rice wine. Food Science and Biotechnology, 29(6), 805-816. 